Mathématiques et sciences humaines
Mathematics and social sciences

177 | Printemps 2007

Varia

Jean-Michel Laslier, « Le vote et la règle majoritaire. Analyse mathématique de la politique ", Paris, CNRS éditions, 2004

Jean-Michel Laslier, "Le vote et la règle majoritaire. Analyse mathématique de la politique ", Paris, CNRS éditions, 2004

\title{
Olivier Hudry
}

\section{OpenEdition \\ Journals}

Édition électronique

URL : http://journals.openedition.org/msh/3936

DOI : $10.4000 / \mathrm{msh} .3936$

ISSN : $1950-6821$

Éditeur

Centre d'analyse et de mathématique sociales de l'EHESS

Édition imprimée

Date de publication : 7 mai 2007

Pagination : 156-158

ISSN : 0987-6936

Référence électronique

Olivier Hudry, « Jean-Michel Laslier, « Le vote et la règle majoritaire. Analyse mathématique de la politique », Paris, CNRS éditions, 2004 », Mathématiques et sciences humaines [En ligne], 177|

Printemps 2007, mis en ligne le 07 mai 2007, consulté le 23 septembre 2020. URL : http:// journals.openedition.org/msh/3936 ; DOI : https://doi.org/10.4000/msh.3936 


\section{ANALYSE BIBLIOGRAPHIQUE}

Jean-François LASLIER, Le vote et la règle majoritaire. Analyse mathématique de la politique, CNRS éditions, Paris, 2004, 208 pages.

Le livre de M. Balinski [1] n'est pas le seul consacré aux problèmes de vote. L'heure étant aux préoccupations électorales, continuons avec un autre ouvrage portant sur les mêmes thèmes. Mais la présentation est ici bien plus mathématique, comme l'indique le titre. sections!:

Le livre est organisé en huit chapitres équilibrés, eux-mêmes subdivisés en

1. «!Attendus méthodologiques et vue d'ensemble!» («!Une théorie de la démocratie en acte!», «!Modèles comportementaux!», «!Le paradoxe de la participation!», «!Une théorie normative!», «!Contenu de l'ouvrage!», «!Leçons pour la philosophie politique!», 12 pages)!;

2. «!Justifier la règle de la majorité!» («!Questions d'opinions, d'intérêts, de délégation!», «!Théorème du jury de Condorcet!», «!Portée générale du théorème du jury!», «!Compétition électorale!», 28 pages)!;

3. «!Modèles unidimensionnels!» («!L'électeur médian!», «!Incertitude et ambiguïté en une dimension!», «!Application!: choix d'un taux de taxe!», 20!pages)!;

4. «!Modèles multidimensionnels!» («!Paradoxes de la séparabilité!», «!Vote spatial!», «!“Chaos” majoritaire!», «!Ensemble découvert!», «!Ensemble couvrant minimal!», 24!pages)!;

5. «!Le vote dans les comités!» («!Paradoxes de la manipulabilité!», «!Vote sophistiqué!», «!Règle de Borda!», «!Règle de Condorcet-Young!», 22 pages)!;

6. «!Le vote populaire!» («!Modèles probabilistes!», «!Ambiguïté stratégique!», 20!pages)!;

7. «!Le respect des minorités!» («!Représentation proportionnelle!», «!Représentation partisane!», «!Minorités sous la règle majoritaire!», 22 pages)!;

8. «!La présidentielle française!» («!Le système à deux tours!», «!Une expérience de vote par approbation!», «!La géométrie de la présidentielle de 2002!», 26 pages).

Un index ( 8 pages en doubles colonnes) et une bibliographie (10 pages, 174 références) complètent ces chapitres.

Ce livre est issu des cours de troisième cycle donnés par l'auteur, directeur de recherche au CNRS et enseignant à l'École polytechnique, sur les modèles de l'économie politique et sur la théorie des choix collectifs en démocratie. Il s'adresse à un lectorat, chercheurs ou étudiants, dont les connaissances requises incluent un certain goût pour les mathématiques (probabilités, analyse, théorie des jeux, etc.) en général et pour la modélisation mathématique en particulier. L'auteur avertit d'ailleurs dans la partie 1.4 que certains concepts développés dans le texte (il cite le chapitre 4) peuvent être très abstraits.

Le premier chapitre présente l'ouvrage et le contexte dans lequel il s'inscrit!: l'étude de «!la démocratie [...] à travers une institution particulière qui est le vote!», et plus particulièrement la règle majoritaire. 
Celle-ci fait l'objet du chapitre 2, avec lequel on entre dans le vif du sujet, notamment grâce au théorème du jury de Condorcet. Une interprétation de la règle majoritaire en termes de compétition électorale y est proposée, qui servira de fil conducteur à l'exposé.

Le modèle unidimensionnel adopté dans le chapitre 3, certes par trop simplificateur pour décrire une situation réelle mais commode pour cette même raison de simplicité, fait l'hypothèse que les entités entre lesquelles on doit choisir peuvent être caractérisées par un seul paramètre. En faisant en outre l'hypothèse que les préférences des électeurs sont unimodales et en supposant enfin que le nombre d'électeurs est impair (pour éviter les ex æquo), le théorème de l'électeur médian nous indique alors qu'il existe une unique médiane des modes individuels et que cette médiane est un vainqueur de Condorcet. Ce modèle fait ensuite l'objet de variantes, par exemple liées à la présence d'informations incomplètes ou défectueuses. Le chapitre se termine avec une illustration, traitée de façon approfondie, sous la forme d'une application au choix d'un taux de taxe.

L'unicité de la dimension est remise en cause dans le chapitre 4. La multidimensionnalité est génératrice de situations paradoxales!: paradoxe des trois référendums et paradoxe d'Ostrogorski par exemple. Des solutions aux problèmes rencontrés sont proposées, fondées sur la règle majoritaire!: l'ensemble des candidats non couverts et l'ensemble couvrant minimal. On retrouve là des ensembles que l'auteur avait déjà étudiés dans un livre précédent [2], consacré à ce qu'il est convenu d'appeler les solutions de tournois (voir la recension [3] qui lui a été consacrée dans un numéro antérieur de Mathématiques et Sciences humaines).

Le chapitre 5 focalise l'attention sur le vote dans les comités, c'est-à-dire dans des situations pour lesquelles le nombre d'électeurs est relativement faible et où donc chaque voix compte (cette situation s'oppose à ce qui est appelé «!vote populaire!» dans le chapitre 6). L'hypothèse de vote sincère peut alors être remise en cause. De nouveaux paradoxes peuvent en ce cas voir le jour, liés à la manipulabilité (que le théorème de Gibbard et Satterthwaite rend inévitable sous de classiques hypothèses), comme l'illustre un exemple appliqué à la règle de Borda. Celle-ci est par ailleurs caractérisée par ses propriétés normatives, tout comme la règle de Condorcet-Young.

Dans le chapitre 6, on suppose que le nombre de votants est grand, ce qui a pour conséquence que l'importance marginale d'une seule voix est faible. Des modèles probabilistes, comme ceux décrits par P.!Coughlin [4], sont envisagés pour prendre en compte des perturbations agissant sur les réponses des électeurs aux propositions des partis. Est aussi abordé le cas où les candidats tiennent des discours ambigus, intentionnellement ou non.

La question de la représentation des minorités fait l'objet du chapitre 7. Trois thèmes y sont traités!: la représentation proportionnelle, avec les problèmes liés aux arrondis (les nombres de sièges à pourvoir doivent être entiers!!), par exemple le paradoxe dit de l'Alabama!; la représentation partisane et la représentation idéologique, dont certains aspects renvoient le lecteur à l'électeur médian du chapitre 3!; enfin une remise en cause de l'idée selon laquelle des partis uniquement motivés par le pouvoir sont amenés à favoriser la majorité au détriment des minorités.

Le dernier chapitre se concentre sur les élections présidentielles en France et en propose une fort intéressante analyse. Celle-ci inclut l'expérience menée par l'auteur et ses collègues lors de l'élection présidentielle de 2002 sur l'application du vote par 
approbation. Ce mode de vote consiste à laisser les électeurs voter simultanément en faveur de tous les candidats dont ils approuveraient l'élection!; le candidat recueillant le plus de voix est élu. L'expérience a été effectuée dans six bureaux de vote pendant le premier tour de l'élection de 2002. Les résultats de cette expérience, minutieusement analysés dans le chapitre!8, permettent une compréhension beaucoup plus fine des positions politiques de l'électorat que celle induite seulement par les reports de voix supposés entre les deux tours d'une élection classique, dans la mesure où les choix multiples exprimés par les électeurs fournissent des corrélations plus riches entre les candidats. L'étude des résultats conduit l'auteur à des conclusions globales comme l'existence d'une gauche et d'une droite mais non d'un centre, ou encore que la vie politique française ne se réduit cependant pas à ce seul axe d'explication et possède bien un caractère multidimensionnel.

Un enseignement que l'on peut tirer de ce livre est que, en matière de vote comme ailleurs, le recours aux mathématiques est indispensable pour comprendre en profondeur les phénomènes observés. Cependant, ce genre de présentation formelle du vote reste peu développé en France et la littérature classique sur le sujet est plutôt anglo-saxonne. - ce titre, l'ouvrage de Jean-François Laslier, en proposant une introduction à une théorie normative des élections, vient combler une lacune regrettable. Écrit avec soin, couvrant un large domaine de la théorie du vote (théorème du jury de Condorcet, paradoxes électoraux, analyse des comportements des jurés, théorème de l'électeur médian, compétition électorale, ambiguïté stratégique, etc.), il constitue de ce fait, d'ores et déjà, une référence sur le sujet pour les chercheurs ou les étudiants francophones intéressés par une approche mathématique des sciences sociales.

O. Hudry

[1] Michel Balinski, Le suffrage universel inachevé, Belin, Paris, 2004, 335 pages.

[2]!Jean-François Laslier, Tournament Solutions and Majority Voting, Berlin, Heidelberg, New York, Springer, 1997.

[3] Olivier Hudry, Analyse bibliographique du livre de J.-F. Laslier!: Tournament solutions and majority voting, Berlin, Springer, 1997, Mathématiques et Sciences humaines 141, 1998, p. 73-76.

[4] Peter J. Coughlin, Probabilistic voting theory, Cambridge, Cambridge University Press, 1992. 